biological markers. Using unbiased magnitude-based hierarchical clustering of biomarker expression levels, we isolated a patient subset $(n=9)$ with distinctively heterogeneous patterns of expression of the 17 biological parameters, compared to the other $(n=101)$ patients who were more homogeneous. The smaller subgroup had significantly higher levels of MIF, CCL2, CCL19 and CXCL10, but the larger subgroup had stronger associations between biological parameters and SLEDAI-2k, based on leave-one-out cross-validated regression analysis. In this subgroup, when we constructed a time-dependent regression model, compared to the equivalent time-agnostic regression model, the biological parameters had significantly stronger predictive power for disease activity, suggesting a timedependent relationship. To disentangle the effect of magnitude versus temporal correlation, we used dynamic time-warping analysis to align longitudinal clinical and laboratory profiles. This revealed a further subset $(n=69)$ in whom a timedependent regression model showed significantly stronger associations between biological parameters and disease activity, despite no significant difference in simple magnitude. This subgroup was characterised by lower rates of flare, lower disease activity and lower damage scores, suggesting that this patient cluster is highly clinically meaningful.

Conclusions: Using aggregated longitudinal clinical data and samples, we demonstrated significant subgroups of time-dependent relationships between disease activity and biological markers among patients with SLE. These results imply the association between biological parameters and disease activity may exist in a multi-dimensional time-dependent pattern. Longitudinal SLE data presents potential opportunities to identify patient-stratifying biomarker patterns that are concealed when time is not considered. This finding has significant implications for the design of SLE biomarker studies.

Disclosure of Interest: None declared

DOI: 10.1136/annrheumdis-2018-eular.2034

\section{SAT0034 POSSIBLE INVOLVEMENT OF BAFF AND MATRIXMETALLOPROTEINASE-9 IN THE ACTIVATION OF MONOCYTES OF PATIENTS WITH PRIMARY SJÖGREN'S SYNDROME}

K. Yoshimoto, K. Suzuki, T. Takeuchi. Keio University School of Medicine, Tokyo, Japan

Background: B cell activating factor belonging to TNF family (BAFF) is well known as a factor which regulates proliferation, differentiation and survival of $B$ cells, and plays a pivotal role in the pathogenesis of primary Sjögren's syndrome (pSS). In our previous study, we found that BAFF significantly enhanced IL-6 production by pSS monocytes and the amount of IL- 6 produced by BAFF-stimulated monocytes was positively and significantly correlated with the expression level of a BAFF receptor (BR3). These data collectively suggest that the BAFF signalling through BR3 is involved in activation of monocytes to promote production of inflammatory cytokines, such as IL-6. Matrix metalloproteinase-9 (MMP-9) is well known as one of the enzymes involved in degradation of extracellular matrix (ECM) and mainly produced by activated T cells and monocytes. It has been reported that the concentration of MMP-9 in saliva was significantly higher in pSS patients as compared to healthy controls $(\mathrm{HC})$. Therefore, it is conceivable that MMP-9 is involved in the pathogenesis of pSS through degradation of ECM of salivary glands, which consequently results in decrease in saliva, one of the clinical manifestations of pSS.

Objectives: To explored the relationship between BAFF and MMP-9 in the pathogenesis of pSS.

Methods: Peripheral monocytes from pSS patients $(n=37)$ and $H C(n=19)$ were prepared by using CD14 +microbeads and cultured in vitro in the presence or absence of recombinant human soluble BAFF (rhsBAFF) for 96 hours. The amounts of IL- 6 and MMP-9 in the culture supernatants were measured by ELISA. Signal transduction pathways were investigated by exposing rhsBAFF-stimulated pSS monocytes to several inhibitors against NF-кB (BAY11-7082 and BAY117085) and PI3 kinase (LY294002). FACS analysis of whole blood samples was performed to investigate the expression levels of BR3 and MMP-9 in monocytes. The expression level of MMP-9 was also analysed by quantitative RT-PCR (qPCR). Serum levels of BAFF and MMP-9 were measured by an electrochemiluminescence assay.

Results: Serum levels of BAFF and MMP-9 in pSS patients were significantly higher than those of $\mathrm{HC}$, and the levels showed positive and significant correlation. FACS analysis of whole blood samples demonstrated that MMP-9 was mainly expressed in monocytes and that the expression level was significantly higher in pSS than in HC. ELISA and qPCR revealed that stimulation of pSS moncytes with rhsBAFF drastically enhanced the expression of MMP-9 as compared to normal monocytes. Remarkably, the amount of MMP-9 produced by the cells was positively and significantly correlated with the expression level of BR3 in pSS monocytes, suggesting that BAFF-signalling is involved in the production of MMP9 by the cells. Moreover, the elevated production of MMP-9 was significantly suppressed by specific inhibitors against NF- $\kappa \mathrm{B}$ and $\mathrm{PI} 3$ kinase in a dose dependent manner.

Conclusions: The present study suggests that BAFF stimulates monocytes through BR3 to promote MMP9 production and may contribute to ECM degradation. Our study also suggests that $\mathrm{NF}-\kappa \mathrm{B}$ and $\mathrm{PI} 3$ kinase are involved in the pathway.

Disclosure of Interest: None declared

DOI: 10.1136/annrheumdis-2018-eular.4149

\section{SAT0035 THE EFFECTS OF VISFATIN, RESISTIN AND IL-17 ON SYNOVIAL FIBROBLASTS FROM DIFFERENT RHEUMATIC DISEASE BACKGROUNDS}

K. Frommer ${ }^{1}$, S. Rehart ${ }^{2}$, M. Sauerbier ${ }^{3}$, U. Müller-Ladner ${ }^{1}$, E. Neumann ${ }^{1}$. ${ }^{1}$ Dept of Rheumatology and Clinical Immunology, Campus Kerckhoff-Klinik, JustusLiebig-University Giessen, Bad Nauheim; ${ }^{2}$ Dept. of Orthopaedics and Trauma Surgery, Agaplesion Markus Hospital; ${ }^{3}$ Dept. of Plastic, Hand and Reconstructive Surgery, BGU Frankfurt, Frankfurt, Germany

Background: Although rheumatoid arthritis (RA) and psoriatic arthritis (PsA) have several features in common, they also possess distinct differences. We hypothesised that RA and PsA synovial fibroblasts (SF), known key effector cells in the pathophysiology of inflammatory arthritis, differentially respond to various stimuli including adipokines and cytokines and that this may contribute to those differences. For example, IL-17 (also found in synovial tissue) is of particular therapeutic significance in PsA but not as effective in RA. Thus far, IL-17 in its isoform IL-17A has been the major therapeutic target in PsA but IL-17F also plays a role in the IL-23/IL-17 axis of inflammatory diseases.

Objectives: Therefore, we analysed the responses of SF from patients with PsA, RA or no rheumatic disease to IL-17A/F \pm TNF- $\alpha$ and the adipokines visfatin and resistin, which show strong expression in the synovium of inflammatory arthritis.

Methods: SF were isolated from patients with PSA, RA or non-rheumatic disease controls (N), each undergoing joint surgery. PsASF, RASF and NSF were stimu lated with human recombinant IL-17A/F, TNF- $\alpha$, visfatin, and resistin. A neutralising anti-IL-17A antibody was used to verify specificity of the IL-17A effects. Secretion of the proinflammatory cytokine IL-6 was used as the initial readout parameter and was quantified using a commercial immunoassay.

Results: Stimulation with visfatin caused a strong increase in IL-6 secretion in all $S F$ types ( $n=3$ each), while resistin had no effect. Differences in responses were not statistically significant between the SF types studied. IL-17A at concentrations found in serum or synovial fluid did not induce IL-6 secretion in any of the SF. Dose-response curve analysis showed that considerably higher concentrations of IL-17A, which may occur locally in tissue, are required for the induction of IL-6 secretion. An anti-IL-17A antibody abolished the effect, thus showing that the effect is specific for IL-17A. The effects of IL-17A and IL-17F on IL-6 secretion by PsASF could be strongly amplified by a co-stimulation with TNF-a (IL-17A: 5-fold vs 113-fold; IL-17F: 1.7-fold vs 39-fold; TNF- $\alpha$ alone: 12-fold). The effects were stronger for IL-17A than for IL-17F with or without TNF co-stimulation. No effect of IL-17F alone was observed on NSF $(n=1)$.

Conclusions: SF from RA and PsA patients were not differentially affected by the adipokines visfatin and resistin or IL-17A when used at serum or synovial fluid concentrations suggesting inflammatory cells to be the primary target of anti-IL-17 therapy. In its use as a therapeutic target, the attribute of IL-17F affecting PSASF would potentially increase the beneficial effects.

Acknowledgements: This work was supported by an unrestricted educationa grant from Celgene $\mathrm{GmbH}$.

Disclosure of Interest: K. Frommer Grant/research support from: unrestricted educational grant from Celgene $\mathrm{GmbH}, \mathrm{S}$. Rehart: None declared, M. Sauerbier: None declared, U. Müller-Ladner: None declared, E. Neumann: None declared DOI: 10.1136/annrheumdis-2018-eular.2691

\section{SAT0036 NICOTINE PROMOTES MMP-3 AND RANKL SECRETION THROUGH OVEREXPRESSED NICOTINIC ACETYLCHOLINE RECEPTOR A7 IN RHEUMATOID ARTHRITIS FIBROBLAST-LIKE SYNOVIOCYTES}

J.-D. Ma' , Y.-S. Mou' ${ }^{2}$, T. Yan ${ }^{2}$, J. Jing ${ }^{1}$, Y.-Q. Mo ${ }^{1}$, Y.-L. Chen ${ }^{1}$, L. Dai ${ }^{1}$.

${ }^{1}$ Rheumatology, Sun Yat-Sen Memorial Hospital, Sun Yat-Sen University;

${ }^{2}$ Zhongshan School of Medicine, Sun Yat-Sen University, Guangzhou, China

Background: Smoking has been reported not only an established environmenta risk factor for developing rheumatoid arthritis (RA), but also a predictor of radiographic progression. Nicotine, the major constituent of cigarette smoke, has been demonstrated inhibitory effect on proinflammatory cytokines through its receptor nicotinic acetylcholine receptor $\alpha 7(\mathrm{AChR} \alpha 7)$ in RA fibroblast-like synoviocytes (FLS). However, its effects on other function of RA-FLS remain elusive. 